\title{
Spectral Astrometry Mission for Planets Detection
}

\author{
D.J. Erskine, J. Edelstein
}

This article was submitted to SPIEs Astronomical Telescopes and Instrumentation, Waikoloa, HI, August 22-28, 2002

\section{August 9, 2002}

U.S. Department of Energy

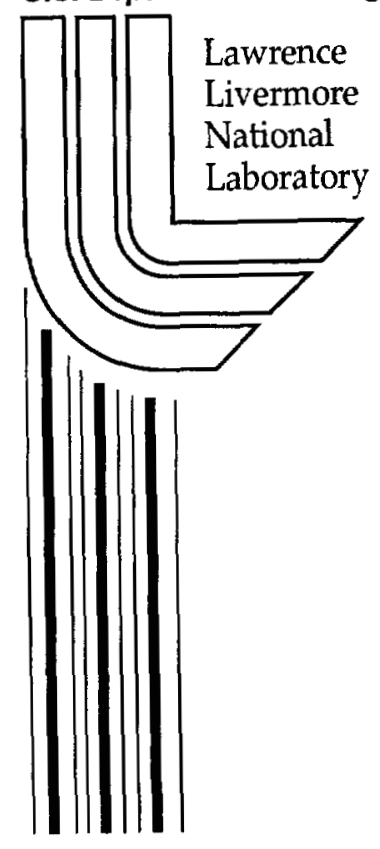




\section{DISCLAIMER}

This document was prepared as an account of work sponsored by an agency of the United States Government. Neither the United States Government nor the University of California nor any of their employees, makes any warranty, express or implied, or assumes any legal liability or responsibility for the accuracy, completeness, or usefulness of any information, apparatus, product, or process disclosed, or represents that its use would not infringe privately owned rights. Reference herein to any specific commercial product, process, or service by trade name, trademark, manufacturer, or otherwise, does not necessarily constitute or imply its endorsement, recommendation, or favoring by the United States Government or the University of California. The views and opinions of authors expressed herein do not necessarily state or reflect those of the United States Government or the University of California, and shall not be used for advertising or product endorsement purposes.

This is a preprint of a paper intended for publication in a journal or proceedings. Since changes may be made before publication, this preprint is made available with the understanding that it will not be cited or reproduced without the permission of the author.

This work was performed under the auspices of the United States Department of Energy by the University of California, Lawrence Livermore National Laboratory under contract No. W-7405-Eng-48.

This report has been reproduced directly from the best available copy.

Available electronically at http://www.doc.gov/bridge

Available for a processing fee to U.S. Department of Energy And its contractors in paper from

U.S. Department of Energy

Office of Scientific and Technical Information

P.O. Box 62

Oak Ridge, TN 37831-0062

Telephone: (865) 576-8401

Facsimile: (865) 576-5728

E-mail: reports@adonis.osti.gov

Available for the sale to the public from

U.S. Department of Commerce

National Technical Information Service 5285 Port Royal Road Springfield, VA 22161

Telephone: (800) 553-6847

Facsimile: (703) 605-6900

E-mail: orders@ntis.fedworld.gov

Online ordering: http://www.ntis.gov/ordering.htm

OR

Lawrence Livermore National Laboratory

Technical Information Department's Digital Library

http://www.llnl.gov/tid/Library.html 


\title{
Spectral Astrometry Mission for Planets Detection
}

\author{
David J. Erskine ${ }^{a}$ and Jerry Edelstein ${ }^{b}$ \\ ${ }^{a}$ Lawrence Livermore Nat. Lab., 7000 East Ave, Livermore, CA 94550 \\ ${ }^{b}$ Space Sciences Lab. at Univ. of Calif., Berkeley, CA 94720-7450
}

\begin{abstract}
The Spectral Astrometry Mission is a space-mission concept that uses simultaneous, multiple-star differential astrometry to measure exo-solar planet masses. The goal of SAM is to measure the reflex motions of hundreds of nearby $(\sim 50 \mathrm{pc}) \mathrm{F}, \mathrm{G}$ and $\mathrm{K}$ stars, relative to adjacent stars, with a resolution of $2.5 \mu$-arcsec. SAM is a new application of Spectral Interferometry (SI), also called Externally Dispersed Interferometry (EDI), that can simultaneously measure the angular difference between the target and multiple reference stars. SI has demonstrated the ability to measure a $\lambda / 20,000$ white-light fringe shift with only $\lambda / 3$ baseline control. SAM's structural stability and compensation requirements are therefore dramatically reduced compared to existing long-arm balanced-arm interferometric astrometry methods. We describe the SAM's mission concept, longbaseline SI astrometry method, and technical challenges to achieving the mission.
\end{abstract}

Keywords: Astrometry, Externally Dispersed Interferometry, Spectral Interferometry, Exoplanet Search

\section{INTRODUCTION}

We describe a space-based differential astrometry mission concept for measuring exo-solar planet masses. The mission concept, called the Spectral Astrometry Mission or SAM, has as its goal the detection of the motions of hundreds of nearby $(\sim 50 \mathrm{pc}) \mathrm{F}, \mathrm{G}$ and $\mathrm{K}$ stars, relative to adjacent stars, with a resolution of $2.5 \mu \operatorname{arcsec}(\mu ")$. SAM is based on the new application of an existing interferometric technique called Spectral Interferometry (SI). The SAM SI instrument can simultaneously measure target and reference stars. Light collected by telescopes, separated by a long-baseline, is combined and measured by SI's hybrid of an unbalanced-arm interferometer and a classical spectrometer. Because the SI technique has demonstrated ${ }^{1,2}$ visible phase shifts resolution of $\lambda / 20,000$ with a baseline control of only $\lambda / 3$, the requirements for SAM's large-scale structural stability or compensation are dramatically reduced compared to existing balanced-arm, single-target interferometric astrometry methods such as those proposed for space interferometry mission ${ }^{3}$ (SIM). The SAM is compact, using $50 \mathrm{~cm}$ telescopes separated by a $2-\mathrm{m}$ 'flexible' baseline. The SAM mission concept offers a promising new path for space-based differential astrometry.

\subsection{The Search for Extra-solar Planets}

The search for extra-solar planets is one of the most visible areas of science today. ${ }^{4,5}$ Ground based measurements of radial stellar velocity reflex have led to the discovery of scores of planets. Important questions regarding the statistical distribution of planet masses and orbital axis remain because the radial velocimetry methods are inherently biased toward the detection of large mass planets with small axes, and because the methods establish only lower planet-mass limits due to undermined orbital inclination. In particular, key questions remain regarding the population of Earth like planets within habitable zones and the population of Jupiter like planets at large distances. It is unclear whether our kind of planetary system is typical or atypical. These issues have profound impact upon the predicted evolution of habitable planets and their atmospheres.

Further author information:

D.J.E.: E-mail: erskine1@llnl.gov, Telephone: 19254229545

J.E.: E-mail: jerrye@ssl.berkeley.edu, Telephone: 15106420599 


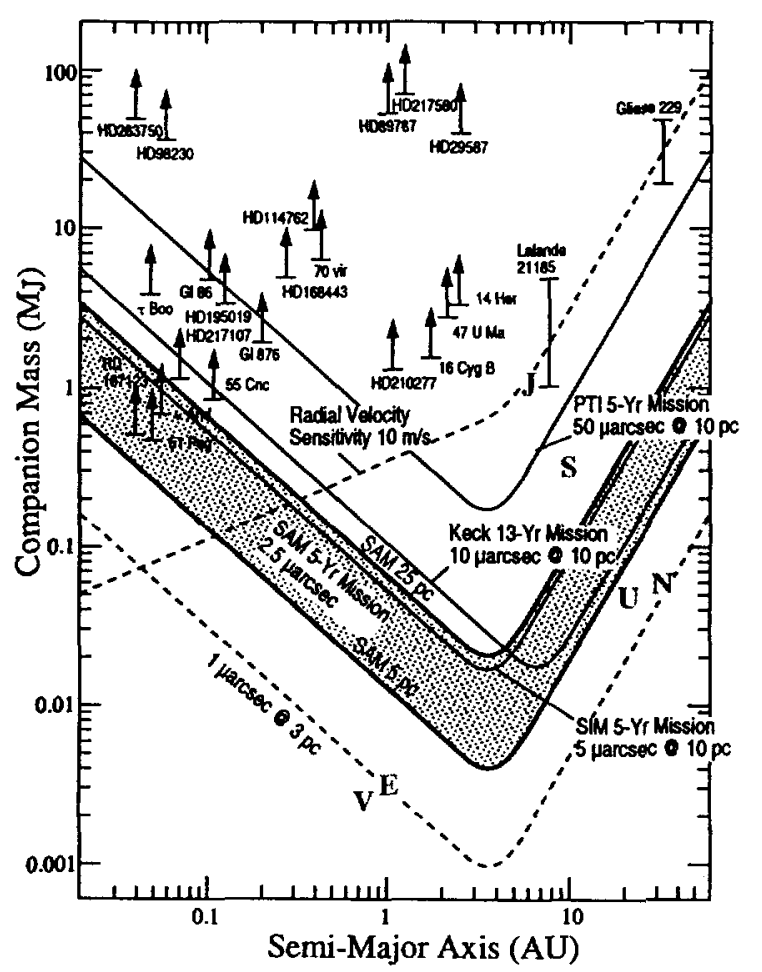

Figure 1. Discovery space. Data taken from SIM report. ${ }^{3}$ Shaded area shows SAM sensitivity from 5-25 pc.

The limitations of radial-velocimetry measurements have inspired efforts to find new methods of directly measuring exo-planets. One method being actively pursued for space-missions is wide-angle astrometry to measure a star's circular reflex motion due to the presence of a planet(s), (such as the SIM concept). The wide-angle astrometry method uses long baseline interferometry (LBI) to measure the positional shift of a target star's centroid relative to other reference stars. By making repeated measurements over time of the target's differential position, the actual reflex orbital motion can be mapped, the orbital inclination estimated, and the planetary mass derived given the target mass due to the stellar type.

The observations required for planetary population investigations are to survey for Earth-like and larger planets around hundreds of Sun-like stars, and to directly determine the masses of the detected planets. The science requirements propagate into inter-related observatory and instrument requirements. The desired range for planet mass and axis measurements determines the minimum ( $\sim 2-3$ months) and maximum measurement interval ( $\sim 5$ years) and so establishes require- ments for mission life and number of exposures per target. The planet mass and axis, together with the target space distribution, establish the astrometric resolution requirements and, taken together with the required number of target measurements, determines the instrument flux sensitivity requirements. The target distribution is derived from the 3-dimensional space distribution (luminosity function) of Sun-like stars (here taken as $\mathrm{F}, \mathrm{G}$, and $\mathrm{K}$ dwarfs).

The relationship between several of these parameters is shown in "discovery space" in Fig. 1. The astrometric resolution needed to detect planets of given orbital axes and masses at specific distances are shown. We conclude that an intermediate, $2.5 \mu$ " resolution, sufficient to detect the reflex motion of an Earth-like planet at $1.2 \mathrm{pc}$, will provide a sufficient sampling of the discovery space to obtain essential science data. A summary of the SAM mission concept parameters are shown in Table 1.

\subsection{Existing Method: Balanced Long-Baseline Interferometry (e.g. SIM)}

We briefly describe the LBI astrometry method used by $\operatorname{SIM}^{6}$ for planet finding. SIM uses a long-baseline balanced-arm interferometer. Light from two collectors at the baseline ends is interfered. The optical path lengths of the two input arms must be precisely matched so that their difference (called the delay $\tau$ ) is nearly zero. In this balanced-path configuration, the fringe phase $(\phi=\tau / \lambda)$ does not vary strongly with wavelength $(\lambda)$. The method requires exquisite dimensional stability or characterization of the large optical paths because as $\tau$ departs from 0 the fringes become unresolvable. For example, the SIM $^{6}$ plans a $7 \mathrm{~m}$ baseline rigid truss to mount open-beam optics, and uses multiple control and metrology loops to stabilize or measure the entire optical path with a total error budget of 340 pico-m, or $\lambda / 1500$ at $5000 \AA$. Limiting dimensional tolerances to this precision over multi-meter baselines is extremely challenging, stretching the state of the art, and budgets.

With this method, a minimum of three separate or sequentially interferometer baseline measurements are required to obtain the relative angular position of the target to two reference stars. This is because balanced LBI techniques cannot distinguish between multiple sources if their starlight is superimposed within a single common path. The inability to use a common path occurs because different objects within the field will generate a similar spectral fringe pattern ${ }^{7}$ that cannot 


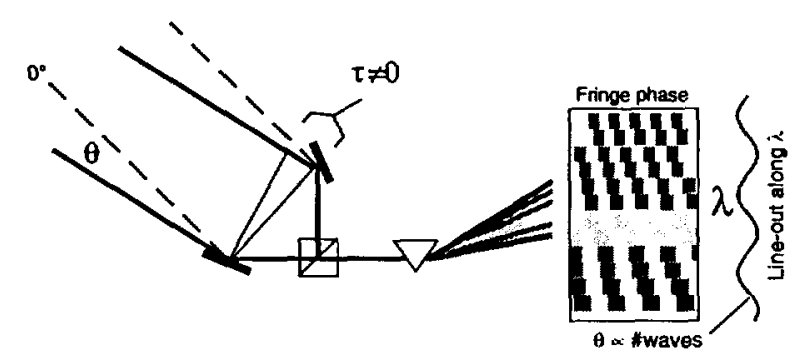

Figure 2. Long baseline measurement of an object off-axis at angle $\theta$ creates an unbalanced interferometer having nonzero $\tau$, producing fringes that vary along the wavelength direction with a density proportional to $\theta$.

be accurately distinguished. Some conventional LBI instruments use up to a few tens of wavelength channels to help retain fringe visibility under parasitic dispersion effects, however this is insufficient to distinguish among multiple simultaneous objects.

\subsection{SAM Method: Unbalanced Long-Baseline Spectral Interferometry}

\subsubsection{System Description}

The SI astrometry system (see Fig. 2) is a long-baseline unbalanced-arm, white-light interferometer that samples the delay $\tau$ (corresponding to illumination angle) between its two apertures over a large number of phase cycles by using dispersion to separate the spectrally varying fringes emanating from a Michelson interferometer. By inserting a reference absorption cell in the beam, a low spatial frequency Moiré pattern results on the detector because of the heterodyning of the two high spatial frequency spectra. This heterodyning relieves the need for a high-resolution spectrometer and expands the measurement range of $\tau$, effectively increasing the angular field of view for a given detector pixel size. Multiple stars can be viewed simultaneously in the enlarged field of view. Each star has a different Moiré pattern that will beat with the other stars pattern. Phase stepping techniques provide robust and accurate determination of the beat pattern phase. Simultaneous multiple reference star observation eliminates the dependence of the baseline length and rotation angle knowledge from the phase determination. Common mode changes in the pathlength of the multiple stars are unimportant, dramatically reducing the stability requirements of the instrument for the large common-path baseline distances.

\subsubsection{Fringe Formation}

Light from two collectors at the baseline $B$ ends are joined in a Michelson interferometer. The optical path difference, $\tau$, of the two inputs are made intentionally large (e.g. many thousands of wavelengths, $\lambda$ ). In this, unbalanced-path configuration the fringe phase varies strongly with $\lambda$. The light from the interferometer is imaged to the slit of a conventional spectrograph and the overlapping phases in the interfered light are dispersed. The sinusoidal fringes form a spectrally dependent comb transmission function,

$$
T(\nu)=(1 / 2)[1+\cos (2 \pi \tau \nu)],
$$

(where $\nu=1 / \lambda$ in $\mathrm{cm}^{-1}$ ), that modulates (multiplies) the input spectrum. The spectral dispersion allows measurement of the Michelson fringes.

\subsubsection{Simultaneous angle measurement}

An essential characteristic of SI is its ability to measure several stars simultaneously. The SI can be used to measure the source position angle to the baseline, $\theta$, because $\theta$ effects the total inter-arm delay by

$$
\delta \tau=B \sin \theta \sim B \theta,
$$

and therefore the density of fringes per unit of frequency. Hence, for small $\theta$, the target angle is proportional to, and uniquely determines, the spatial fringe frequency across the bandwidth $(\Delta \nu)$ of the spectrograph:

$$
\theta=(\# \text { fringes }) /(B \Delta \nu) .
$$

This is significant because multiple targets, observed simultaneously at different position angles, will generate multiple spatial frequencies that can be readily separated using post-detection Fourier decomposition (see Fig. 3 for a laboratory demonstration of this phenomena). Differential angular measurement between simultaneous targets are robust to changes over the long baseline because light from all of the stars travel simultaneously through this common path. Therefore, common optical element changes such as detector distortions, focal spot shape, and temporal changes in the stellar spectrum or intensity are negated.

\subsubsection{Importance of simultaneous} measurements

SI's capability to simultaneously measure stars has several very important ramifications.

(1) The need for long-path baseline stability is relieved because the differential angle measurements are not affected by deviations (typically environmental) in the 
common-conduit path.

(2) The determination of differential position angle can be made independent of the value of the baseline length if two reference stars are simultaneously measured against the target. Hence, $\delta \theta$ can be measured to high accuracy even if the baseline shifts within or between measurements (see Eq. 11, which shows $\delta \theta$ is independent of B).

(3) Determining $\delta \theta$ can also be made independent of absolute baseline rotation-angle if three reference stars are simultaneously measured against the target because the new reference coordinates allow for a recursive estimation of roll errors. Consequently, the need for critical control or measurement of observatory rotational pointing is decoupled from the interferometric result.

(4) Simultaneous measurements of both target and reference stars present an observing efficiency advantage compared to sequential observations.

\subsubsection{Spatial heterodyning of angle measurement}

Because the spectral fringe frequency increases with position angle, the maximum measurable angle is proportional to the resolution of the spectrograph (and the pixel size of the detector). In order to measure large angles, while keeping the spectral resolution $R=\lambda / \delta \lambda$ modest and the consequent payload size, mass and complexity low, a Moiré or heterodyning process is used. The light, after interference, is passed through a wavelength reference cell (comb) that imprints (multiplies) a known spectrum against the input spectrum. The reference spectrum imprints the light with thousands of stable spectral fiducials. The sinusoidal fringes due to angular position (Eqs. $1 \& 2$ beat against the features of the reference spectrum to form a Moiré pattern on the detector. These Moiré patterns rotate in phase with position angle in the same way as its underlying spectral fringes.

\subsubsection{Beat phase measurement} For spectral astrometry, the position-angle induced Moiré would produce a unique fringe pattern, which manifests pattern for one star is measured simultaneously with the Moiré pattern for another star. The phase of the beats formed between the two patterns is a highly sensitive measurement of the differential angle $\delta \theta$ between the two stars. The beat phase, is independent of drifts in the common path delay. The $\delta \theta$ measurement is insensitive to the absolute position angle because, while the underlying fringe pattern frequency changes with $\theta$, the beat pattern frequency does not. Because the Moiré patterns are large compared to the spectral fringes
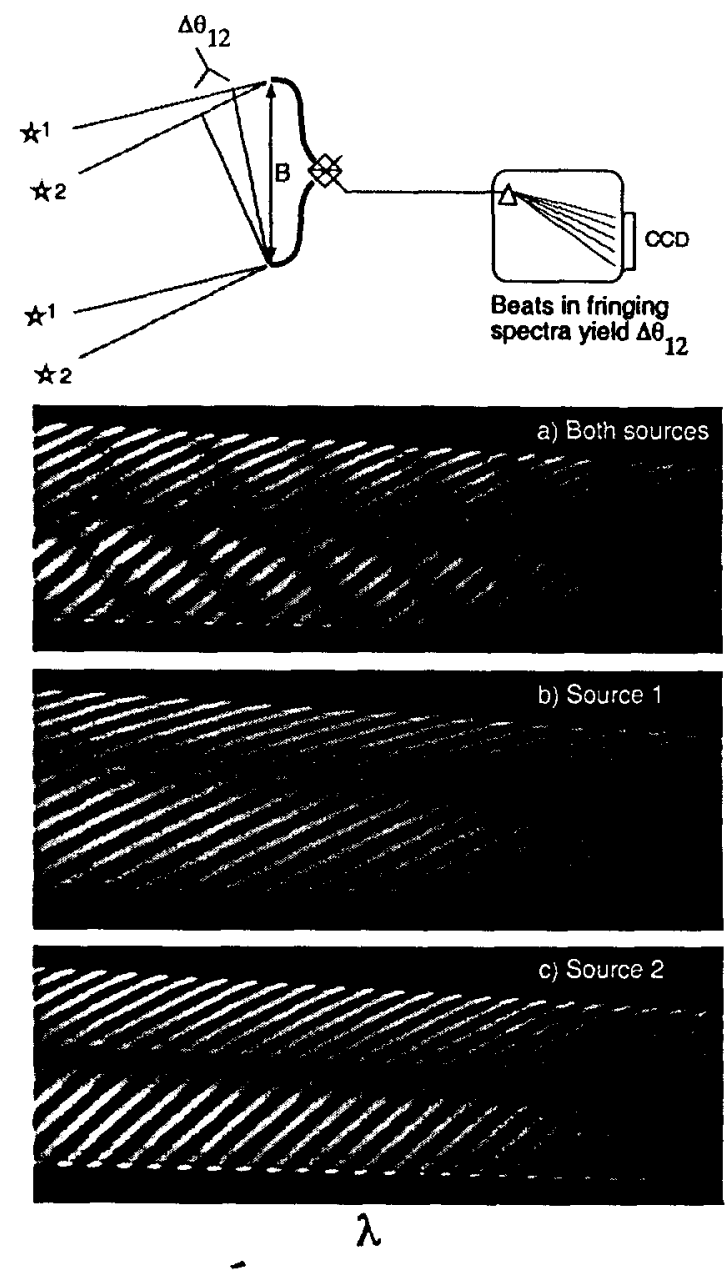

Figure 3. Laboratory data demonstration of multi-object SI. Two stars observed by the spectral interferometer each beats when summed at the detector. The white light fringe patterns shown were obtained using the prototype SI modified to have a baseline $B$, and two white light pinhole sources simulating stars, both together (\#a with beats) and separately (\#b, \#c). Pinholes were separated by $0.5^{\circ}$ with $\mathrm{B}=6 \mathrm{~mm}$ and spectrum bandwidth $350 \AA$ over $5400 \AA$. 
themselves, the system is quite insensitive to the optical quality of the common path system. For example, while variable or aberrant spectrometer resolution or focus may change or reduce the Moiré pattern contrast, it will not degrade the structure of the pattern itself.

\subsubsection{Phase Stepping improvements}

We implement a phase-stepping method to provide accurate phase determination and to allow for a straightforward data-analysis algorithm to eliminate fixed pattern instrument noise and delay drifts up to $\lambda / 3$. We arrange for the interferometer delay $\tau$ to linearly vary slightly along the spectrometer input slit axis (called the vertical axis) by tilting one interferometer mirror across the spectral dispersion plane. The resulting slanted interferometer comb pattern can be analyzed along the vertical axis at each spectral channel. Thus, the real and imaginary components of the fringe phase can be derived by using a series of self-referenced phase measurements of the Moiré fringe within a single exposure. Although this cross-dispersion sinus phase variation itself allows for phase determinations, additional phase stepping is still advantageous because it removes fixed pattern noise such as detector flat-field variations. Hence, we introduce delay modulation with a PZTtransduced mirror by taking four exposures with delay steps of about $\lambda / 4$. A proven, sophisticated phase algorithm ${ }^{8}$ does not require knowledge of the phase stepping size. The step size is found as part of a minimization procedure that takes advantage of the independence of the fringing wavelength channels.

\section{DEMONSTRATION OF SPECTRAL INTERFEROMETRY PERFORMANCE}

We have demonstrated a compact spectral interferometer in our laboratories to have a phase resolution of $\Delta \tau=27$ picometers or $\lambda / 20,000 .^{1,2}$ This SI was designed for a Doppler velocimetry application. Theory of the Moire fringe formation and phase stepping data processing technique common to both the Doppler and astrometry applications is in Refs. 8-10. Figure 5 shows the SI. Light from a source fiber passes through an iodine vapor cell used as a spectral reference. Hence the light now consists of both the potentially Doppleraffected source spectra and the stationary iodine absorption spectrum (5000-5800 $\AA$ ). The light transits an unbalanced Michelson interferometer, with a $\tau \sim 1$ $\mathrm{cm}$ delay, and is then dispersed by a commercial $R=20$ $k$ spectrograph to a CCD detector. Fringes were observed with various sources including sunlight, bright

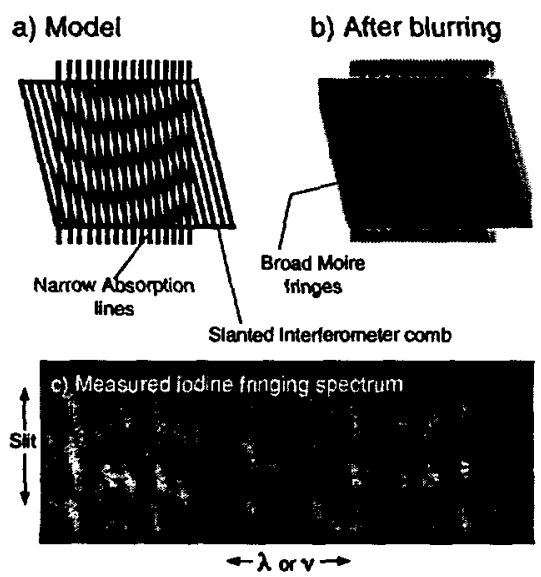

Figure 4. Demonstration of Moiré or heterodyning effect on actual iodine absorption spectrum c) recorded by SI prototype for a fixed delay of about $1.1 \mathrm{~cm}$. Each delay creates a unique Moiré pattern which can be used to determine the delay to $27 \mathrm{pm}$ precision with a 0-6 cm range. a) Model. b) Blurring due to spectrograph slit does not prevent detection of Moiré fringes.

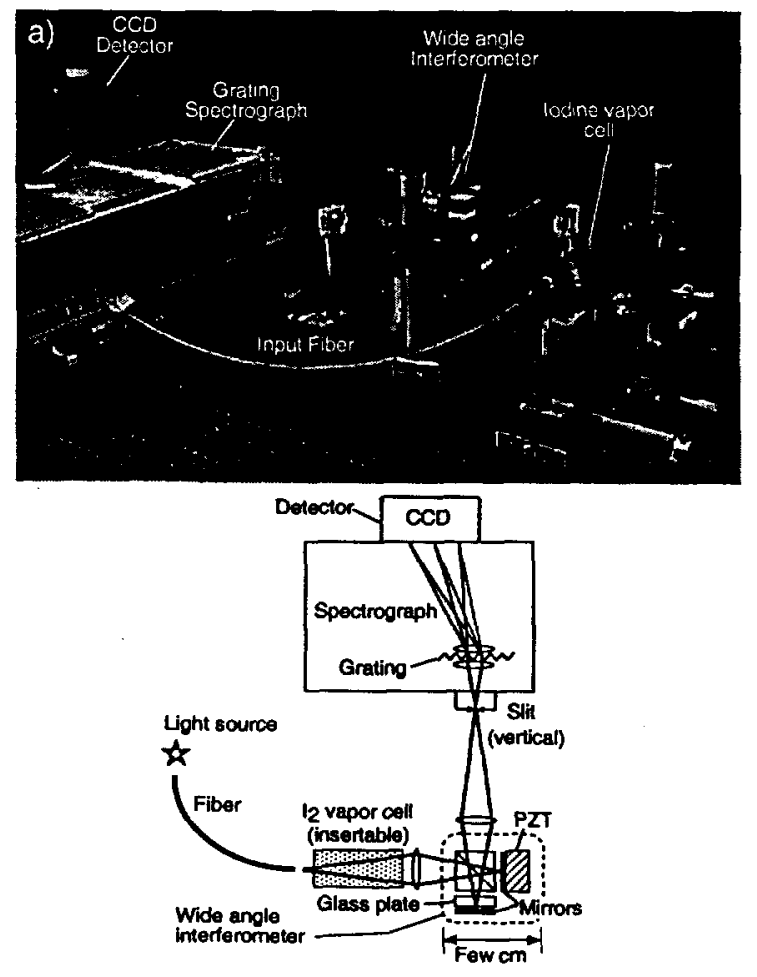

Figure 5. Photo and schematic of EDI (SI) prototype used to take solar, iodine and bright starlight velocimetry data. 
starlight, and simulated stationary starlight consisting of a backlit bromine vapor cell. Figure 4 shows SI's Moiré fringes resulting from beating an iodine reference spectrum against the comb from a $\tau=1.1 \mathrm{~cm}$ unbalanced interferometer. The relative shift between a stationary "bromine stellar source" and the reference iodine cell spectra were measured repeatedly ${ }^{1,2}$ to find an instrument noise-limited performance of 0.76 $\mathrm{m} / \mathrm{s}$, which is equivalent to $\lambda / 20,000$. Although the velocimetry and astrometry data analysis techniques slightly differ, the $\lambda / 20,000$ performance result can be directly applied to our astrometry application.

The Doppler SI suffered a long period ( 2 week) zeropoint velocity drift $(\sim 4 \mathrm{~m} / \mathrm{s})$, a small magnitude that is quite remarkable given that the instrument was not thermally or optically stabilized. Regardless, astrometry requires repeated precision across long measurement intervals. Fortunately, these zero-point drifts are immaterial to our astrometry because the SAM approach uses a differential method that obtains a new zero-point template spectra for each measurement.

The broadband nature of SI is a primary reason for the order of magnitude fringe-shift precision improvement compared to conventional monochromatic interferometers. The presence of thousands of wavelength channels, each having independent, evenly sampled fringe phase, means that systematic phase errors are statistically diminished. In fact this type of multichannel spectral method has been applied, using 32 channels, to improve group-delay fringe tracking for the ground based Navy Prototype Optical Interferometer. ${ }^{11}$

\section{THEORY}

\subsection{Spectral beats for astrometry}

The angular difference $\Delta \theta$ between two objects in the SI is detected through the presence of beats in the fringing spectra vs. $\nu$ (Fig. 3). First consider a single target. As $\tau$ increases, the fringing spectra twists (linearly increasing phase angle change $\Delta \phi$ versus $\nu$ ) because $\Delta \phi$ is wavelength dependent, $\Delta \phi=\Delta \tau / \lambda$. (Our analysis, which determines the absolute $\tau$ that creates a given fringing spectrum, is not confused by integer fringe skips, as is a monochromatic system. Now consider two temporally incoherent broadband sources \#1 and \#2. Assume that $\theta_{1}$ and $\theta_{2}$ are widely separated so that $\left(\tau_{1}-\tau_{2}\right) \nu>1$, i.e. so that at least 1 fringe exists over the spectrograph bandwidth. Then these two fringe patterns will not be confused, as we show. Since both star light 1 and 2 travel through the same reference spectrum cell, they each make a similar fringing spectrum but having different amounts of twist. Fringing spectra are represented by complex waves, whose phase and magnitude vs. $\nu$ represent the phase and visibility for a given spectral channel. Let $W_{1}(\nu)$ and $W_{2}(\nu)$ be fringing spectra for stars 1 and 2 measured alone, and $W_{0}(\nu)$ the spectra for the reference cell alone (i.e. the instrument at $\theta=0, \tau=0$ ). It can be shown that the fringing spectra are related to the intrinsic spectrum $W_{0}(\nu)$ by a phasor

$$
W_{k}(\nu)=W_{0}(\nu) e^{i 2 \pi \nu \tau_{k}}
$$

which applies the spiral twist. The addition of two different amounts of twists on the same fringing spectrum creates beats in net amplitude and phase, depending on the relative intensity a of the sources:

$$
\begin{aligned}
W_{1}+\alpha W_{2} & =W_{0}(\nu) e^{i 2 \pi \nu \tau_{1}}+\alpha W_{0}(\nu) e^{i 2 \pi \nu \tau_{2}} \\
& =W_{0}(\nu) e^{i 2 \pi \nu \tau_{1}}\left(1+\alpha e^{i 2 \pi \nu \Delta \tau}\right) .
\end{aligned}
$$

The second term contains the beats in both phase and magnitude. The number of beats across the bandwidth $(\mathrm{BW})$ is

$$
n=(\Delta \tau / \lambda)(B W / \lambda)
$$

and is proportional to the difference in source angle,

$$
n=\Delta \theta(B / \lambda) \cos \theta(B W / \lambda) .
$$

The expected angular displacements created by a planet tugging a star will produce fringes from observation to observation that change by much less than a single revolution of twist. Because the whole number of beats changes so slightly, we choose to measure the phase of the beats for a fixed $n$ to determine $\Delta \theta$. The use of spectral beats for astrometry has been theoretically discussed by James, Kandpal and Wolf. ${ }^{12,13}$ Our method differs by the inclusion of the reference cell that greatly increasing the maximum measurable delay, and by the use of phase stepping techniques that very precisely measure the beats in both amplitude and phase.

\subsection{Independence from Baseline}

The SI measures the fraction angular position of the target relative to the spacing between the two reference stars. Using small angles (the conclusion is valid for larger angles), each star's delay is due to its angle plus the common pathlength offset $\mathrm{C}$ :

$$
\tau_{1}=B \theta_{1}+C ; \tau_{2}=B \theta_{2}+C ; \tau_{3}=B \theta_{3}+C .
$$




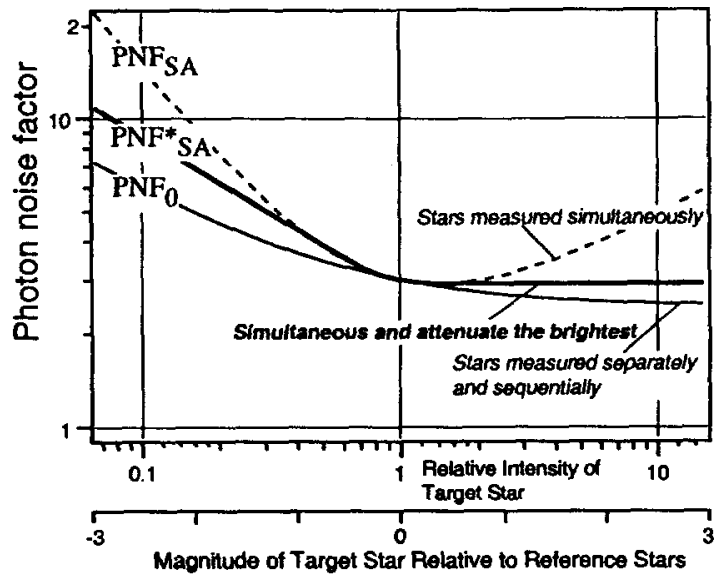

Figure 6. Photon noise component of angular precision vs intensity of target star relative to two other reference stars.

If the target is \#2 and the reference \#1 and \#3, then the baseline is

$$
B=\left(\tau_{1}-\tau_{3}\right) /\left(\theta_{1}-\theta_{3}\right)
$$

and

$$
\delta \theta=\left(\theta_{2}-\theta_{3}\right)=\left(\theta_{1}-\theta_{3}\right) \frac{\left(\tau_{2}-\tau_{3}\right)}{\left(\tau_{1}-\tau_{3}\right)} .
$$

Hence, two or more simultaneous reference stars remove the baseline value $B$ from determination of $\delta \theta$.

\subsection{Photon-limited Sensitivity}

The single star photon limited angular resolution of a SI interferometer is

$$
\delta \theta=\frac{\sqrt{2} \lambda}{\pi B V} \mathrm{PNF}
$$

where $V$ is fringe visibility $(0.2$ to $<1$ typical) and the photon noise factor

$$
P N F=\sqrt{1 / N_{p h}}
$$

where $N_{p h}$ is the number of photons. For multiple stars measured separately the PNF is described by

$$
P N F_{0}=\sqrt{1 / N_{1}+1 / N_{2}+1 / N_{3}}
$$

which is plotted in Fig. 6 as thin curve for a case where the target star magnitude is varied relative to two reference star intensities. In comparison, for measuring 3 stars simultaneously, the PNF is described by

$$
P N F_{S A}=\sqrt{N_{1}+N_{2}+N_{3}} \sqrt{1 / N_{1}^{2}+1 / N_{2}^{2}+1 / N_{3}^{2}}
$$

which is plotted in Fig. 6 as dashed curve. It is beneficial to make all 3 stars equal intensity. The bold curve, $P N F_{S A}$, shows when the brightest stars of a triad are attenuated to minimize the PNF. To maximize sensitivity, the range of target and reference star flux should be limited. This can be accomplished by target selection (as we have done for our target estimates) or by relative attenuation which, in theory, could be instrumentally implemented. Note that measuring the stars separately means measuring them sequentially. For a fixed total integration time, $N_{p h}$ will be reduced $3 \times$, raising $P N F_{0}$ by $\sqrt{3}$. Hence sequential and simultaneous observations produce similar photon limited behavior when the stars are of similar intensity.

\subsection{Multi-Star Target Statistics}

We have examined the Tycho and Hipparcos stellar catalogs to find candidate target stars with nearby reference stars suitable for differential astrometry measurements. We identified all dwarf stars of type F, G and $\mathrm{K}$ as possible targets. We counted as candidates the number of target stars with magnitude $\leq m_{v}$, within a distance $\leq d$, and with 3 or more reference stars within a certain angular distance to the target star. The catalogs completeness begins to limit the population for $m_{v}<10$. Our results are summarized in the Table 2 . Our analysis shows that an SI with a $0.5^{\circ}$ to $1^{\circ} \mathrm{FOV}$ with sensitivity to detect $m_{v}<9$ or 10 should be able to observe the desired number of science targets. Our candidate values are not estimates, but actual catalog counts. Not all of these stars may be ideal targets for SAM science however. We have not yet tested the relative separation and orthogonal alignment, parameters that can effect the astrometric uncertainty.

\section{PRELIMINARY INSTRUMENT DESIGN}

Figure 7 shows our straw-man adaptation for the SAM SI instrument. Two telescopes, separated by a baseline distance $B$, collect coherent broadband light beams from distant targets. Given SI's demonstrated $\lambda / 20,000$ wavefront precision, then the $2.5 \mu$ " angular resolution goal can be achieved with a 2 meter baseline.

\subsubsection{Telescope size}

Sensitivity requirements determine the telescope size and bandpass. From our prior analysis (Eq. 12), we estimate that a total $\sim 700$ million events must be detected to achieve $2.5 \mu$ " resolution with a $2-\mathrm{m}$ baseline assuming a $50 \%$ fringe visibility and reference and target stars with similar intensity. These events may be 


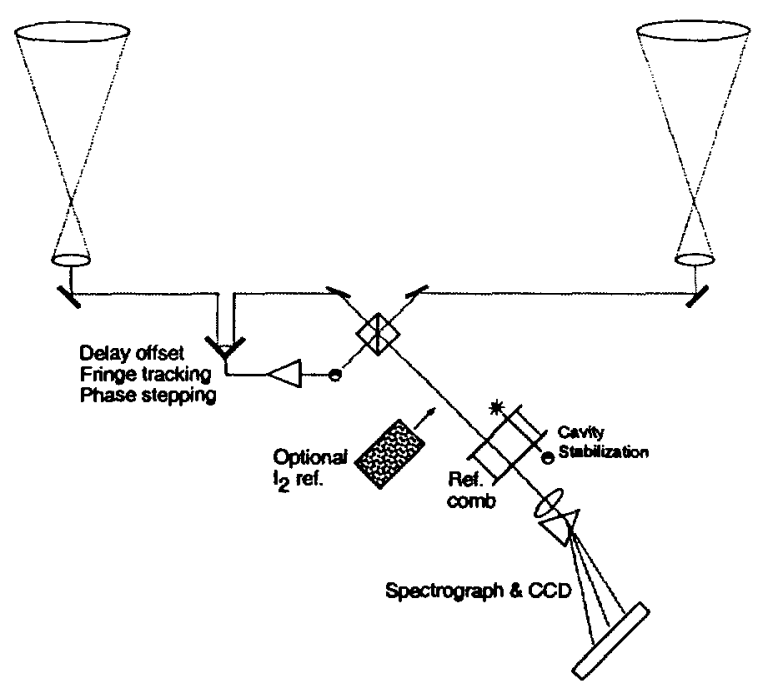

Figure 7. Simplified schematic of a spectral interferometer used in simultaneous astrometry of multiple sources. Light from sources are collected by two input telescope systems (not shown) and injected into the ends of left and right conduits separated by a baseline B. The left \& right beams are interfered at the beam splitter and dispersed to a CCD detector. The angle $\theta$ of each source creates a time delay between the left \& right arms, which manifests fringes vs. wavelength across the recorded spectrum having density (1/spacing) proportional to source angle. Multiple sources travelling the same common path through the conduit create beats in spectrum. These measure the differential source angle $\Delta \theta_{12}$ independent of conduit length. Coarse $(\lambda / 3)$ fringe tracking prevents fringe skips. A spectral reference is used to heterodyne dense fringe spacing to a lower density resolvable by spectrograph. The reference could either be an iodine absorption cell or a laser stabilized interferometer.

accumulated in any series of independent observation. To observe 150 targets four times per year then each observation may accumulate a total integration time of $\sim 25,000 \mathrm{~s}$, assuming an observational efficiency of $50 \%$. To measure orthogonal angular positions on the sky, two observations per star are needed unless a second, two-telescope SI is included in the payload design. We presume SAM includes a pair of SI's for both observational efficiency and redundancy. Assuming reasonable component efficiencies $\left(7^{*} \mathrm{M} 93 \%, \mathrm{G} 80 \%, \mathrm{Q}\right.$ $70 \%$, BS $50 \%, 4^{*}$ T $95 \%$, fiber coupling $50 \%$ ), the total throughput is $\sim 8 \%$. Presuming a 50-cm telescope aperture with $70 \%$ obscuration, we derive that a 600 $\AA$ bandpass is required to observe a $9^{\text {th }}$ magnitude G2 star.

\subsubsection{Focal plane fiber collector}

At the focal plane, four fiber collectors are shuttled to a fixed position to collect each star's light. We envision that co-aligned star-trackers per telescope actuate an "anti-jitter" mirror system that stabilizes starlight in the correct focal plane position for beam collection. The four fiber paths from each telescope must be combined into a single beam or fiber optical conduit in a common mode mixer (e.g. via a pinhole or single mode fiber). We envision using single-mode joiners similar to those commercially available.

For each star, the path from the first telescope surface to the point of beam combination is non-common. Consequently, great care must be taken to control or measure relative shifts between each of the stars' optical path. The focal plane collection and non-common path treatment represent the primary technical challenges to be mastered. We describe methods of resolving these issues below, and note the happy fact that the dimensional control issue must be resolved over the size scale of an individual telescope, not over the multimeter baseline scale.

\subsubsection{Flexible Baseline Conduit}

A long baseline conduit carries mixed light from each focal plane to the interferometer. The conduit can consist of flexible optical fiber because using simultaneous reference stars negates any changes in the commonpath baseline. Once the individual fibers have been forced to share the same wavefront by, say travel through a pinhole or short length of single mode fiber, then any subsequent path shares a common wavefront and can be of any type such as a large diameter multimode fiber or an open beam. Our straw-man uses multimode fiber for the long common path between the input optics and interferometer. Flexible conduit allows greater freedom in the mechanical design and layout of the payload and eliminates the need for a large rigid (subwave) baseline truss. While single mode fibers produce a more controlled fringe phase at the spectrometer, multimode fibers tend to have a larger transparency bandwidth.

\subsubsection{Gross Optical Delay}

An optical delay line trombone is actuated over many $\mathrm{cm}$ in one arm to allow for an adjustable non-zero delay offset, which is also equivalent to a FOV pointing offset that allows for compensation of large-scale observatory pointing errors. We anticipate launching the fiber light to a small, collimated open beam prior to entering the delay line. 
During each individual exposure, the arm path length must be stable to $\lambda / 3$ or net fringe visibility will be diminished. This can be accomplished using a conventional, small-motion optical-path stabilizing $(\lambda / 3)$ actuator in one of the two arms. A huge advantage of SI is that the relative stellar angle precision is de-coupled from the phase precision $(\phi)$ so pathlength stabilization $<\lambda / 3$ is not necessary. (The SIM method must achieve $\lambda / 1500$ phase precision because, in this case, $\phi$ directly sets the angular resolution.) The small-motion actuator also can be used to provide $\sim \lambda / 4$ phase stepping per exposure. The optical-path actuator(s) fine positioning is controlled (typically piezoelectrics) by a fringe phase sensor.

\subsubsection{Field of View}

The light from both arms are combined with a beam splitter and passed through a reference cell. The maximum angle that can be measured in a single observation is $\theta_{\text {max }}=\tau_{\text {coh }} / B$ where $\tau_{\text {coh }}$ is the coherence length of the reference cavity. For an iodine cell $\left(\tau_{\text {coh }} \sim\right.$ $6 \mathrm{~cm}$ ) and a $2 \mathrm{~m}$ baseline $\theta_{\max }$ is $1.7^{\circ}$. However, the field of view of the SAM will also be constrained by the input optics design and the statistics of the distribution of the target and reference stars. We illustrate the choice of either a stabilized interferometric cavity cell, and a gas absorption cell. Interferometric cavities tend to have much higher fringe visibility and coherence lengths than gas cells and, in principle, can have their fringe spacing actively tuned to provide the optimum comb function for observations. The gas cell, can provide an absolute fiducial wavelength grid that can be used to calibrate the tunable cavity or to extract a wavelength-calibrated high resolution spectrum from and individual star.

\subsubsection{Spectrometer and CCD}

A spectrometer's slit is at the focus of the reference cell output beam. The $\mathrm{R}=20,000$ spectrometer disperses the fringes to a wide ( $4000 \mathrm{pxl}) \mathrm{CCD}$ detector that covers the $600 \AA$ bandwidth centered at $5000 \AA$. The CCD need not be as tall (128 pxl) because the nondispersion axis is used to read low spatial frequency Moire pattern phase.

Exposures per target are taken in sequence for each $\lambda / 4$ phase. Maximum exposure time will be bound by the depth of the CCD well and by the ability of the SI system to maintain fringe stability. The phase stepping algorithm removes high frequency fixed pattern noise and fringe patterns from the data image and eventually returns the Moiré beat complex phase.

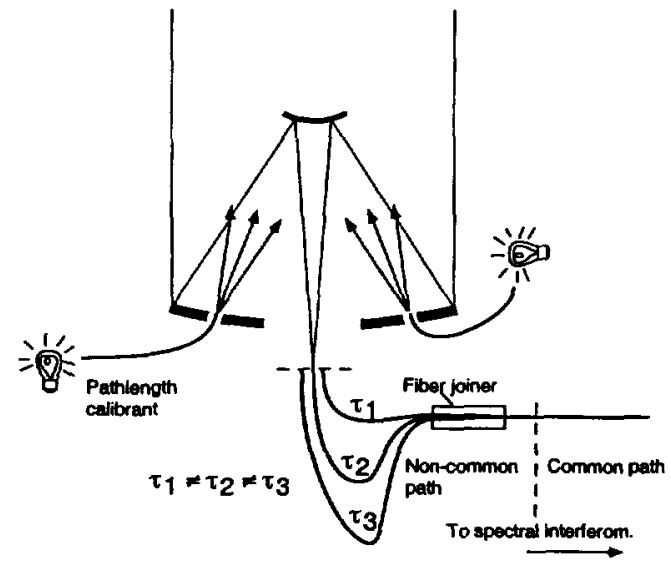

Figure 8. Schematic of white light calibration of noncommon path delay. Light from fiber creates fringes due to the deliberately differing lengths of the input fibers. For a Cassegrain, a tripod of fibers determines the XYZ location of each fiber input.

\section{FURTHER DESIGN CONSIDERATIONS}

\subsection{Reference cells}

In principal, any stable spectrum can be used for a fiducial spectral reference, such as an $I_{2}$ absorption spectrum or the periodic spectrum provided by a stabilized interferometer or cavity (e.g. Michelson, Fabry Perot or Lyot cell). The reference cell's spectral comb stability must match the desired SI system fringe resolution. The technology to meet cell stability requirement is tractable. Gas absorption cells (e.g. $\mathrm{I}_{2}, B r_{2}$ ) have $>\lambda / 20,000$ stability for $\sim 6 \mathrm{~cm}$ cavities. A FabryPerot (FP) has been demonstrated in a 1-cm cell with $\sim \lambda / 20,000$ stability using a using semiconductor-laser stabilization (Connes ${ }^{14}, 15$ ). In principle, this FP stabilization technique should be applicable to much longer coherence lengths.

\subsection{Internal phase calibration system}

Light from each star must be joined into a common mode in a way that does not cause uncorrectable changes to the relative pathlength for each star. That means that the entire uncommon path from the first telescope surface to the mixed fiber must be stable or measurable to the wavefront resolution requirement of $\lambda / 20,000$. This requirement is the key technical challenge to the SAM's implementation. 
Our approach to the problem is to directly measure any differences in the individual star beam pathlengths in situ with the same SI used for the stellar measurements. White light is inserted by one of three narrow fibers affixed about the primary surface (Fig. 8). A spherically expanding wavefront propagates through the optics to each collecting fiber. The fibers have pathlengths that differ by more than the light coherence length to avoid data confusion between the fibers. The calibrant light creates a fringing spectrum and the fibers' differential pathlengths are determined using the same apparatus and method as for starlight. The exact location of the emitting fibers does not matter, since offsets generate a pathlength error linear in $\theta$ are eliminated by using two reference stars. Using three fibers in a tripod allows all 6 dimensions of differential rotation and translation to be determined. Then only non-linear temporal mirror figure distortions between the fiber locations must be controlled or calibrated. The calibration would occur separately for each input arm and the three fibers would be energized sequentially. The source need not be exactly white, and could be a high-brightness photo-diode so that calibration exposures would be rapid.

\subsubsection{Telescope induced phase errors}

Telescope illumination effects and temporal optical excursions could induce differential phase errors to the star beam paths. Since only differences in pathlengths between the individual stars matters, then only higher order effects are generally important. For example, a non-spherical wavefront at the fibers focus is, in itself, not detrimental, because as the telescope pointing wanders all the fibers will move over the focal spot in the same way. Secondly, wavefront differences do not matter if they are linear in position, because such terms can be shown to be equivalent to a change in baseline $B$ to form an effective baseline $B^{\dagger}$, an uncertainty eliminated by using two simultaneous reference stars (Eqs. 9-11). Only a secondary-induced, quadratic wavefront distortion can cause significant differential beam path changes. An offending quadratic term in the data difference,

$$
\left(\operatorname{Data}_{j}-\operatorname{Data}_{k}\right)=B^{\dagger}\left(\theta_{j}-\theta_{k}\right)+\beta\left(\theta_{j}^{2}-\theta_{k}^{2}\right),
$$

could be eliminated by subtracting instantaneous white light calibration. If the calibrations bracket the stellar exposure in time, then the offense will be cancelled. (However, subtle errors can arise if $\beta$ is varying, such as due to thermal effects.) 
Table 1. SAM Straw-Man Parameters

\begin{tabular}{|c|c|}
\hline \multicolumn{2}{|r|}{ Mission } \\
\hline $\begin{array}{l}\text { Mission Duration } \\
\text { Payload Envelope } \\
\text { Spacecraft Mass } \\
\text { Science Data }\end{array}$ & $\begin{array}{l}5 \text { years } \\
3 \mathrm{~m} \times 3 \mathrm{~m} \times 1.5 \mathrm{~m} \\
600-800 \mathrm{~kg} \text { (approximate) } \\
0.5-1 \text { Gbit /day, unreduced } \\
5-10 \text { observations / orbit @ 6.4 Mbit/orbit }\end{array}$ \\
\hline \multicolumn{2}{|r|}{ Science } \\
\hline $\begin{array}{l}\text { Angular resolution } \\
\text { Fringe Sensitivity } \\
\text { Targets per year } \\
\text { Candidates } M_{v} \leq 9 \text { FGK } \\
\text { Reference stars } \\
\end{array}$ & $\begin{array}{l}2.5 \mu \operatorname{arcsec} \\
\lambda / 20,000 \text { for } 9^{t h} \text { Magnitude G star in } 25,000 \text { sec. } \\
\sim 150 \text { stars } \times 4 \text { observations in orthogonal axes } \\
1000 / 2600 \text { stars within } 50 \text { pc }\left(30^{\prime} / 60^{\prime} \text { FOV }\right) \\
\text { Minimum } 3 \text { per field within } 30^{\prime} / 60^{\prime}\end{array}$ \\
\hline \multicolumn{2}{|r|}{ Instrument } \\
\hline $\begin{array}{l}\text { Telescopes } \\
\text { Field of View (FOV) } \\
\text { Image stabilization } \\
\text { Telescope Focal Plane } \\
\text { (for } 30^{\prime} \text { FOV) } \\
\text { Fiber optics } \\
\text { Beam splitter } \\
\text { Reference cell(s) } \\
\text { Grating Spectrometer } \\
\text { Detector }\end{array}$ & 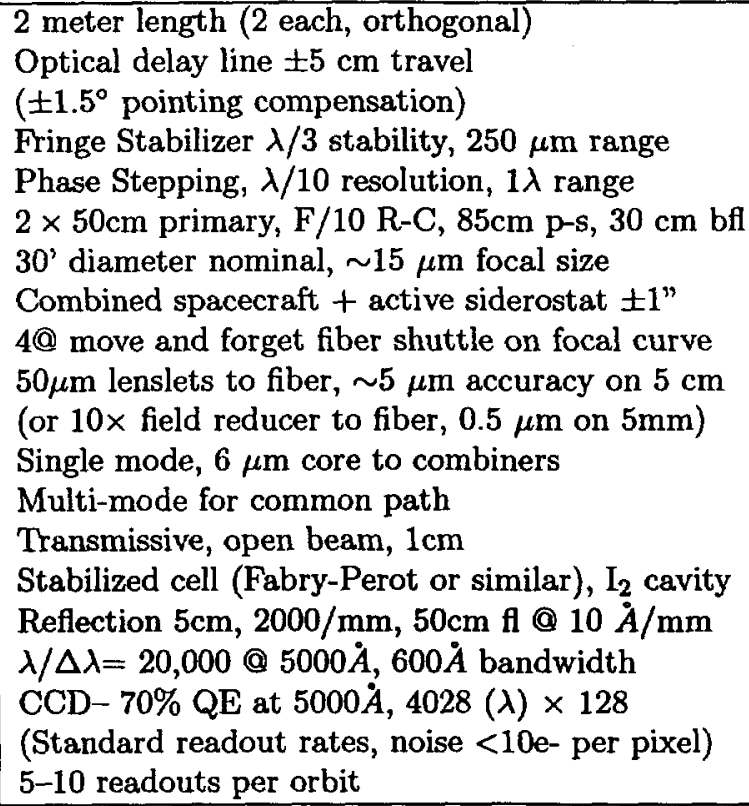 \\
\hline
\end{tabular}

Table 2. a) Number of candidate F, G, K targets with 3 reference stars within a Field of View (FOV). b) Planet mass inducing detectable motion on a $\mathrm{G}$ star by distance to star.

\begin{tabular}{|l|l|l|l|l|}
\hline Target $d \leq(p c)$ & Target $m_{v} \geq(\mathrm{mag})$ & $\begin{array}{l}60^{\prime} \text { FOV } \\
\text { Candidates } \\
(\mathrm{N} \text { stars })\end{array}$ & $\begin{array}{l}\text { 30' FOV } \\
\text { Candidates } \\
(\mathrm{N} \text { stars })\end{array}$ & $\begin{array}{l}\text { Mass of planet at 2 AU } \\
\text { causing 2.5 } \mu^{\prime \prime} \text { motion } \\
\text { of a G star. (Marth }\end{array}$ \\
\hline 50 & $9 / 10$ & $2653 / 3293$ & $1004 / 1415$ & 21 \\
\hline 25 & $9 / 10$ & $370 / 435$ & $116 / 162$ & 10 \\
\hline 10 & 10 & 15 & 5 & 4 \\
\hline
\end{tabular}




\section{ACKNOWLEDGMENTS}

Thanks to John Vallerga for assistance with the manuscript, Pat Jelinsky for statistics of star distribution, and John Mather for fruitful discussions. Work was partially supported by CalSpace/Lockheed, and NASA SARA research grants NAG5-9091 and NAG5-3051. This work was performed under the auspices of the U.S. Department of Energy by the University of California, Lawrence Livermore National Laboratory under contract No. W-7405-Eng-48.

\section{REFERENCES}

1. D. Erskine and J. Ge, "Novel interferometer spectrometer for sensitive stellar radial velocimetry," in Imaging the Universe in Three Dimensions: Astrophysics with Advanced Multi-Wavelength Imaging Devices, W. van Breugel and J. Bland-Hawthorn, eds., ASP 195, p. 501, 2000.

2. J. Ge, D. Erskine, and M. Rushford, "An externally dispersed interferometer for sensitive doppler extrasolar planet searches," PASP , 2002, accepted for publication.

3. http://sim.jpl.nasa.gov/, Oct 2001.

4. D. Lin, P. Bodenheimer, and D. Richardson, Nature 380, p. 606, 1996.

5. A. Cumming, G. Marcy, and R. Butler, Astrophys. J. 526, pp. 890-915, 1999.

6. M. Shao and D. Wolff, SPIE 2477, pp. 228-239, 1995.

7. P. Lawson, "Phase and group delay estimation," in Principles of Long Baseline Stellar Interferometry, P. Lawson, ed., 9, pp. 113-141, 2000. See Fig. 8.2.

8. D. Erskine, "An externally dispersed interferometer prototype for sensitive radial velocimetry: Theory and demonstration on sunlight," PASP , 2002, submitted March 2002.

9. D. Erskine and J. Edelstein, "High- resolution broadband spectral interferometry," in Astronomical Telescopes and Instrumentation, J. Angel, ed., SPIE, Aug 2002. paper 4854-48 of this conference.

10. D. Erskine, "Combined dispersive/interference spectroscopy for producing a vector spectrum," US Patent $6,351,307,2002$.

11. J. T. Armstrong, D. Mozurkewich, L. J. Rickard, D. J. Hutter, J. A. Benson, P. F. Bowers, N. M. Elias, C. A. Hummel, K. J. Johnston, D. F. Buscher, J. H. Clark, L. Ha, L.-C. Ling, N. M. White, and R. S. Simon, "The Navy Prototype Optical Interferometer," ApJ 496, pp. 550-+, Mar. 1998.

12. D. James, H. Kandpal, and E. Wolf, Astrophys. J. 445, pp. 406-410, 1995.

13. H. Kandpal, K. Saxena, D. Mehta, J. Vaishya, and K. Joshi, J. Modrn. Opt. 42, pp. 447-454, 1995.

14. J. Schmitt, P. Connes, and J. Bertaux, "Development of an absolute accelerometer for extra-solar planet detection," Earth, Moon and Planets 81, pp. 83-90, 1998.

15. P. Connes, "Absolute astronomical accelerometry," Astrph. \& Spc. Sci. 110, pp. 211-255, 1985. 